\title{
Estudo da prevalência de endoparasitos em fezes de cães domiciliados na zona norte de Porto Velho, Rondônia, Brasil
}

Study of endoparasites prevalence in stools dogs home in the north zone of Porto Velho, Rondônia, Brazil

Estudio de la prevalencia de endoparásitos en heces de perros domiciliados en el norte de Porto Velho, Rondônia, Brasil

Thiago Vaz Lopes

ORCID: https://orcid.org/0000-0002-3192-1908 Universidade Federal do Acre, Brasil E-mail: Thiagovlopes@hotmail.com

João Gustavo da Silva Garcia de Souza ORCID: https://orcid.org/0000-0002-5899-2490 Centro Universitário Aparício Carvalho, Brasil

Wilyanna Eliza de Souza Morais ORCID: https://orcid.org/0000-0002-6198-5363 Centro Universitário Aparício Carvalho, Brasil E-mail: Wilyannaelisa@gmail.com

Gabriela Burton Matsuno de Almeida ORCID: https://orcid.org/0000-0002-6045-9900

Centro Universitário Aparício Carvalho, Brasil E-mail: Gabriela_burton@hotmail.com

Felipe Muriel Peixoto Rosas ORCID: https://orcid.org/0000-0002-1876-2914 Centro Universitário Aparício Carvalho, Brasil E-mail: Felipe_muriel@hotmail.com

Thaís de Almeida Souza ORCID: https://orcid.org/0000-0002-2722-9601 Centro Universitário Aparício Carvalho, Brasil E-mail: thaís_medvet@hotmail.com

Igor Mansur Muniz ORCID: https://orcid.org/0000-0003-0863-6647 Universidade Federal de Rondônia, Brasil E-mail: igor.mansur@unir.br

Sandro de Vargas Schons ORCID: https://orcid.org/0000-0001-9811-5356 Universidade Federal de Rondônia, Brasil E-mail: sandroschons@unir.br

Fernando Andrade Souza

ORCID: https://orcid.org/0000-0002-9474-9404 Universidade Federal do Paraná, Brasil E-mail: femedvet@yahoo.com.br

\section{Resumo}

É necessária a identificação das endoparasitoses mais comuns, pois algumas podem ser caracterizadas como zoonoses. Dentre as possíveis infecções, destacam-se Ancylostoma spp. e Toxocara canis. Os principais sintomas de infecção observados são desarranjo gastrointestinal, emagrecimento e vômito. Este estudo teve como objetivo identificar as endoparasitoses mais comuns em cães domiciliados na zona norte de Porto Velho-RO, destacando a principal e criando um parâmetro do risco de possíveis zoonoses para a população. Foram coletadas 40 amostras de fezes de cães no período de setembro a novembro de 2019 e feito a análise microscópica utilizando a técnica de Willis e Mollay para preparação das lâminas. Em 40 amostras, 35 foram constadas presença de ovos de helmintos, sendo o mais prevalente identificado o Ancylostoma spp.

Palavras-chave: Endoparasitose; Infecção; Zoonose.

\footnotetext{
Abstract

Identification of the most common endoparasites is necessary, some may be characterized as zoonoses. Possible infections include Ancylostoma spp. and Toxocara canis. The main symptoms of infection observed are gastrointestinal
} 
disorder, weight loss and vomiting. This study aimed to identify the most common endoparasites in dogs domiciled in the north of Porto Velho-RO, highlighting the main one and creating a parameter of the risk of possible zoonoses for the population. Forty dog feces samples were collected from September to November 2019 and the microscopic analysis was performed using the Willis and Mollay technique for slide preparation. In 40 samples, 35 were found to have helminth eggs, the most identified being Ancylostoma spp.

Keywords: Endoparasites; Infection; Zoonosis.

\section{Resumen}

Es necesario identificar las endoparasitosis más frecuentes, ya que algunas se pueden caracterizar como zoonosis. Entre las posibles infecciones, Ancylostoma spp. y perreras Toxocara. Los principales síntomas de infección observados son trastornos gastrointestinales, pérdida de peso y vómitos. Este estudio tuvo como objetivo identificar la endoparasitosis más común en perros domiciliados en el norte de Porto Velho-RO, destacando la principal y creando un parámetro de riesgo de posibles zoonosis para la población. Se recolectaron cuarenta muestras de heces de perro de septiembre a noviembre de 2019 y se analizaron microscópicamente utilizando la técnica de Willis y Mollay para la preparación de portaobjetos. En 40 muestras, se encontró que 35 tenían huevos de helmintos, siendo el más prevalente identificado el Ancylostoma spp.

Palabras clave: Endoparasitosis; Infección; Zoonosis.

\section{Introdução}

As verminoses caninas têm importância para a saúde pública, uma vez que algumas delas são zoonoses. Podendo ser destacada a larva migrans cutânea e larva migrans visceral, sendo estas zoonoses as formas larvais de Ancylostoma spp. $e$ Toxocara canis, respectivamente (Silva et al. 2007; Silva et al. 2017; Machado, Barros \& Bittencourt, 2019). Alguns protozoários presentes no trato gastrintestinal dos cães, também podem acometer os humanos, tais como Giardia spp. e Cryptosporidium spp., que podem causar infecções intestinais em humanos (Labruna et al. 2006; Camassola et al. 2018).

Cães domiciliados que não recebem de forma rotineira medicação vermicida, aliado aos animais de rua, geralmente negligenciados por autoridades quanto a programas de sanidade animal, realizam um papel epidemiológico grande na persistência e propagação dos endoparasitas, que são expelidos através das fezes dos animais, contaminando áreas urbanas. (Prates et al. 2009; Ferreira et al. 2009). Adultos e crianças que convivem com animais, ou frequentam ambientes contaminados pelas fezes, podem infectar-se pela ingestão acidental de ovos e cistos, ou pela migração cutânea de helmintos em sua fase larval, sem nem mesmo ter conhecimento do que pode ter acontecido com elas (Santarém et al. 2004; Oliveira et al. 2009; Mendonça, 2020; Lutinski et al. 2021; Nunes et al. 2018; Youssef et al. 2020).

Após a infecção, normalmente por ovos, os parasitas se deslocam pela circulação sanguínea e tecidos do hospedeiro, até atingirem a forma adulta e se alojarem no intestino delgado, completando assim o chamado Ciclo de Loss (Anderson, 2000).

Os sinais clínicos observados são emagrecimento, desarranjo gastrointestinal, tosse, anemia, enterite, convulsões, vômito e até mesmo pneumonia devido ao deslocamento pulmonar das larvas, verificada com maior ocorrência em animais jovens (Nunes et al. 2000).

O presente estudo teve como objetivo determinar a prevalência de endoparasitas em animais domiciliados na zona norte de Porto Velho-RO.

\section{Material e Métodos}

Foram coletadas amostras de fezes caninas de setembro a novembro de 2019. Para a coleta utilizou-se potes coletores universais, sendo estes entregues aos tutores durante uma visita, na qual ele respondeu um questionário, abordando alguns pontos importantes: número de animais na residência, sexo, idade, número de pessoas que convivem com esses animais, presença de crianças na casa, entre outras informações que servirão para estipular o risco da saúde humana, com base nos endoparasitas possivelmente encontrados nas amostras obtidas. No dia seguinte a visita a equipe do projeto retornou às residências para recolher os coletores, já com as amostras de fezes coletadas pelos tutores, seguindo as orientações passadas na primeira visita. As amostras 
foram mantidas sob refrigeração até o momento do processamento, sendo transportadas em caixas térmicas, até o Laboratório de Parasitologia. Executaram-se coletas periódicas em diferentes residências da zona norte, seguindo o método cego randomizado, até obter no total 40 amostras, optando-se por fezes frescas ou com aspecto de não ressecadas para que fossem evitados falsos negativos.

Para a pesquisa de ovos e oocistos realizou-se as técnicas de Willis-Mollay. O princípio desta técnica é o da flutuação: a fração coletada para exame microscópico é o da superfície, onde a concentração de ovos pode estar aumentada. Confeccionouse uma lâmina por amostra.

Para a realização da técnica de Willis e Mollay mistura-se em um Becker dois gramas de fezes com $20 \mathrm{ml}$ da solução saturada de $\mathrm{NaCl}$, para fluidificação. A solução foi filtrada através da peneira, recoberta com gaze, passando então a suspensão filtrada para um outro Becker, até formar um menisco convexo. Após isso uma lâmina de vidro foi colocada sobre o Becker, com o objetivo de que a lâmina entre em contato com o líquido (menisco convexo). Em seguida, com um descanso de 15 minutos, examinou-se a lâmina no microscópio óptico. Sendo esse um exame microscópico qualitativo direto que permite identificação de ovos e larvas de nematódeos e oocistos de protozoários.

\section{Resultados e Discussão}

As 40 lâminas foram analisadas, das quais 35 amostras foram identificadas com ovos de helmintos. Dentre as lâminas que não apresentaram ovos, os relatos dos tutores indicaram o uso de vermífugo entre os últimos 4 meses. Em relação as amostras com presença de ovos, os tutores de 29 cães relataram fatos como: o não uso de vermífugo no último ano e até mesmo nunca terem vermifugado seu animal. Esse dado representa um total descaso em relação aos cuidados básicos com o cão, além de que algumas dessas helmintoses serem zoonoses, agindo assim com descuido com a própria saúde. Na Tabela 1 observa-se quais foram as espécies encontradas.

Tabela 1. Porcentagem das espécies encontradas em cães domiciliados da zona norte de Porto Velho.

\begin{tabular}{lc}
\hline Espécie & Quantidade de amostras com ovos (\%) \\
\hline Ancylostoma spp & $47.5 \%$ \\
Toxocara canis & $25 \%$ \\
Giardia spp & $2.5 \%$ \\
Ancylostoma spp. e Toxocara canis & $12.5 \%$ \\
Ancylostoma spp e Giardia spp. & $2.5 \%$ \\
\hline
\end{tabular}

Fonte: Autores.

Pode-se observar que a maior incidência foi de Ancylostoma spp, que reforça o estudo realizado em Fortaleza-CE, por Moraes et al. (2018), onde das 40 amostras coletadas a maior porcentagem (38\%) foi da espécie Ancylostoma spp, caracterizando uma prevalência não somente em cães dessa região, mas na espécie canina no geral. Reafirma-se também essa maior prevalência levando em consideração a forma de como seus ovos são dissipados no ambiente.

Nas amostras positivas para Toxocara canis, a segunda maior porcentagem, os tutores não relataram o uso de vermífugo, sendo que essa é uma das principais helmintoses, descritas principalmente em cães jovens, até o sexto mês como relata Gennari (2015), em seu estudo realizado em São Paulo-SP.

O menor índice encontrado (2,5\%), de Giardia spp, diverge com a pesquisa de Funada et al. (2007) que obteve um resultado de $8,5 \%$, sendo relatado como a segunda maior parasitose gastrointestinal.

A endoparasitose múltipla de Ancylostoma spp e Toxocora canis é comum, e já descrita em um estudo realizado por Leite et al. (2004), sendo a mais frequente (3,79\%), e reafirmada por Oliveira et al. (2009) com porcentagem semelhante $(4,0 \%)$. 
A associação com menor resultado, Ancylostoma spp e Giardia spp, foram descritas por Prates (2009) ao descrever uma prevalência de $1,2 \%$.

\section{Conclusão}

O presente estudo pode demonstrar que as infecções gastrointestinais são muito comuns, mesmo em cães domiciliados e reafirma a infecção por Ancylostoma spp como a mais comum em cães na atualidade, sendo a espécie mais identificada das amostras, com uma porcentagem de $47.5 \%$. Por ser uma zoonose leva-se a pensar em como melhorar a vermifugação dos animais com a conscientização dos tutores os persuadindo a agirem de forma correta.

\section{Referências}

Anderson, R. C. (2000). Nematode Parasites of Vertebrates Their Development and Transmission. CABI Publishing.

Camassola, J. et al. (2018). Diagnóstico de Giardia spp., em amostras fecais coletadas nas praças de Pedro Osório, RS. Anais do $10^{\circ}$ Salão Internacional de Ensino, Pesquisa e Extensão da UNIPAMPA: Salão de Pesquisa-Oral, 2(3).

Ferraz, A. et al. (2019). Frequência de Giardia sp. em amostras de fezes de cães e gatos, recebidas no laboratório de doenças parasitárias da UFPEL, entre os anos de 2015 e 2016. Revista Científica Rural, 21(1): 158-164.

Ferreira, M. A. S. (2009). Avaliação de endoparasitos em cães domiciliados, de Abrigo e errantes na cidade de Aracaju - Sergipe. Medicina Veterinária, 3(3): $20-25$.

Funada, F. P. et al. (2007). Frequência de parasitos grastrointestinais em cães e gatos atendidos em hospital-escola veterinário da cidade de São Paulo. Arq. Bras. Med. Vet. Zootec., 59(5): 1338-1340.

Gennari, S. M. (2015). Principais helmintos intestinais em cães no Brasil. Boletim BayerVet, São Paulo, Ano 02 , Edição 08.

Labruna, M. B. (2006). Prevalência De Endoparasitas Em Cães Da Área Urbana Do Município De Monte Negro, Rondônia - Arq. Inst. Biol., São Paulo, 73(2): $183-193$.

Leite, L. C. (2004). Endoparasitas em cães (Canis familiaris) na cidade de Curitiba-Paraná-Brasil. Archives of Veterinary Science, 9(2): 95-99.

Lutinski, J. A. (2021). Parasitoses em Cães domiciliados em um município do Sul do Brasil. Interfaces Científicas - Saúde E Ambiente, 8(3): $151-162$.

Machado, M. O. \& Bittencourt, L. H. F. B. (2019). Levantamento de endoparasitos de cães internados em três centros médicos veterinárias em Cascavel, Paraná. Arquivo Brasileiros de Medicina Veterinária, 2(2): 8-17.

Moraes, P. V. S. (2018). Incidência de Ancylostoma spp e Toxocara spp em cães recolhidos pelo Centro De Zoonoses. Ciência Animal, $28(4)$ : 31-34.

Mendonça, T. O. (2020). Parasitoses em cães domiciliados em um Município de Rondônia, Bioma Amazônia: avaliação quanto ao risco à saúde humana. Dissertação de Pós- graduação pela Universidade Estadual Paulista (UNESP), Faculdade de Ciências Agrárias e Veterinárias, Jaboticabal.

Nunes, H. C. et al. (2018). Prevalência de parasitas intestinais em Cães triados no centro de controle de zoonoses de Gurupi, Tocantins. Revista Cereus, 10(3): 27-37.

Oliveira, V. S. F. (2009). Ocorrência de helmintos gastrintestinais em cães errantes na cidade de Goiânia-GO. Revista de Patologia Tropical, $38(4)$ : 279-283.

Prates, L. (2009). Frequência de parasitos intestinais em cães domiciliados da cidade de Maringá, PR. Arquivo Brasileiro de Medicina Veterinária e Zootecnia, 61(6): 1468-1470.

Santarém, V. A., Giuffrida, R. \& Zanin, G. A. (2004). Larva migrans cutânea: ocorrência de casos humanos e identificação de larvas de Ancylostoma spp. em parque público o município de Taciba, São Paulo. Revista da Sociedade Brasileira de Medicina Tropical, 37(2): 179-18.

Silva, A. S. et al. (2007). Prevalência de Parasitismo em cães domiciliados num bairro de Santa Maria - RS. Revista do Centro de Ciências da Saúde, 33(1): 2731.

Silva, J. C. S et al. (2017). Endoparasitoses em Cães e gatos diagnosticados em São Luís-Maranhão. PUBVET, 11(6): 538-645.

Snak, A. et al. (2019). Ocorrência de parasitos gastrointestinais em cães e estudo de fatores de risco em propriedades rurais da região oeste do Paraná, Brasil. Medicina Veterinária Preventiva UFRPE, 13(3): 391-398.

Youssef, A. G. et al. (2020). Prevalência de Parasitas Intestinais, de Importância Zoonótica, em cães assintomáticos de canis na região de Marília-SP. Brazilian Journal of Development, 6(12): 94718-94727. 